\title{
MERCADO LABORAL Y CRECIMIENTO ECONÓMICO RECOMENDACIONES DE POLÍTICA PARA CHILE
}

\author{
Elías Albagli
}

Este trabajo revisa la relación teórica y empírica entre instituciones y regulación laboral, el funcionamiento del mercado del trabajo y el crecimiento económico. A la luz de las implicancias en eficiencia y bienestar y de las características estructurales del mercado laboral chileno, se proponen medidas para aumentar la eficiencia y la participación laboral bajo una visión que legitima sistemas de protección al trabajo. Entre las principales se encuentran: sustitución del financiamiento de la protección al empleo desde el sistema de indemnizaciones hacia el seguro de desempleo; mayor flexibilidad de horarios y tipos de contratos; crear una regla de ajuste del salario mínimo de acuerdo a indicadores de desempeño macroeconómico; financiar equitativamente el cuidado infantil entre hombres y mujeres; crear sistemas de certificación de capacidades y mayores incentivos a la capacitación por parte de empresas. Las principales mejoras en crecimiento y bienestar vendrían por la vía de aumentos en productividad, participación laboral y mejoramiento del capital humano.

Elías Albagli Iruretagoyena. Ingeniero comercial, Pontificia Universidad Católica de Chile. Magíster en economía financiera, Pontificia Universidad Católica de Chile. Actualmente cursa un doctorado en economía en la Universidad de Harvard. 


\section{Introducción}

$\mathrm{E}$ I funcionamiento del mercado laboral impacta directamente en las decisiones y en el bienestar de todos los individuos. De él dependen la cantidad de gente que está dispuesta a ser parte de la población activa, la rapidez con que se movilizan los factores de producción entre sectores y actividades, la capacidad de la economía de responder a shocks externos y la velocidad con que sus integrantes adquieren capacitación, se adecuan a nuevos procesos e incrementan su productividad. El funcionamiento de este mercado es determinante, por tanto, en el crecimiento económico de los países.

En muchos sentidos, el mercado laboral se rige por las leyes básicas de oferta y demanda. Sin embargo presenta dos particularidades que requieren de un análisis más específico para explicar fenómenos como el desempleo y su persistencia, la evolución de la participación de la fuerza de trabajo y el comportamiento de los salarios, entre otros. La primera se refiere a las características de los participantes: la oferta de trabajo está compuesta de individuos, que difieren en múltiples aspectos, tales como el grado de calificación, experiencia y preferencias. De igual forma, la demanda de trabajo se compone de empresas que requieren trabajadores con habilidades específicas para llevar a cabo sus tareas. Esta heterogeneidad da origen a un proceso de búsqueda o "calce” entre las partes, caracterizado por un flujo constante de trabajadores entre el empleo y el desempleo que implica algún grado de desempleo "friccional”.

La segunda se centra en el marco regulatorio que caracteriza la relación laboral. La gran mayoría de las economías modernas cuentan con regulaciones e instituciones que buscan mejorar las condiciones laborales y proteger los puestos de trabajo —-mediante la regulación de las jornadas, el pago de indemnizaciones por despido, aviso de término de contrato-, velar por el bienestar de la población cesante —a través de seguros de desempleo autofinanciados o estatales, y agencias estatales de búsqueda de empleo- e intervenir en la determinación salarial, ya sea directamente, mediante la fijación de salarios mínimos, o indirectamente mediante la regulación del proceso de negociación colectiva entre trabajadores y empresas.

Sin embargo, este marco regulatorio es señalado frecuentemente como el principal culpable del alto desempleo en algunas economías europeas, porque introduce distorsiones en el proceso de calce. En particular, la literatura suele ligar la pobre capacidad para generar nuevos puestos de trabajo, y por tanto los altos y persistentes niveles de desempleo, a los 
altos costos de despido, los generosos e ineficientes seguros de desempleo y las condiciones de negociación colectiva imperantes en varios países de Europa continental desde mediados de los 70. Este desempeño contrasta con las menores tasas de desempleo en Estados Unidos, y recientemente en Inglaterra, que ostentan mercados laborales menos regulados.

Ante esta situación parece tentador proponer medidas simplistas para mejorar el funcionamiento de los mercados laborales: terminar de raíz con todo tipo de protección laboral ${ }^{1}$. Éste sería, sin embargo, un enfoque equivocado —además de políticamente imposible de implementar-, ya que la protección laboral cumple un rol clave en aumentar el bienestar de los trabajadores. En efecto, éstos valoran no sólo el ingreso que perciben durante su empleo, sino también la estabilidad del mismo, y esta preocupación suya proviene en gran medida de fallas en el mercado del crédito que les impide suavizar su consumo mediante el endeudamiento en períodos de desempleo. Esto es particularmente relevante para los trabajadores de menores ingresos ${ }^{2}$.

Si bien la protección laboral se justifica por las imperfecciones mencionadas, un mal diseño regulatorio e institucional genera incentivos perversos que distorsionan el proceso de calce, lo que afecta al nivel de empleo y a la productividad del mismo, y eso impacta directamente en el bienestar de los trabajadores y en el crecimiento de la economía. El rol de los economistas y encargados de políticas debe enfocarse, por tanto, en corregir dichas distorsiones para lograr un funcionamiento eficiente del mercado laboral dentro de un marco de protección valorado por los trabajadores.

Este trabajo tiene como propósito revisar la teoría y evidencia empírica acerca de la relación entre el funcionamiento del mercado laboral y el crecimiento económico, y de cómo las distintas instituciones laborales afectan a dicho funcionamiento, de manera de abordar las principales debilidades del mercado laboral chileno con una serie de propuestas de alta prioridad, a la luz de sus principales características estructurales.

La estructura del trabajo es la siguiente. En la segunda parte se revisa la conexión teórica y la evidencia empírica entre el funcionamiento del mercado laboral y el crecimiento. En la tercera parte se realiza un diagnóstico de las principales características del mercado laboral chileno, revisando sus instituciones y regulaciones vigentes en un contexto internacional, de manera de identificar los principales focos de mejoría, para plantearse en la

${ }^{1}$ Ver argumento similar en Blanchard y Tirole (2005).

${ }^{2}$ Ver Bertola (2005) para una explicación de otras fallas de mercado que justifican la existencia de esquemas de protección laboral. 
cuarta sección diversas propuestas destinadas a mejorar el funcionamiento del mercado laboral y el crecimiento económico. En la quinta sección presentan las conclusiones.

\section{Instituciones laborales, eficiencia y crecimiento}

\subsection{Teoría}

Son diversos los canales por los cuales la regulación y las instituciones laborales afectan a la eficiencia del mercado de trabajo y, por ende, al crecimiento económico. Entre los principales se encuentran:

\section{La tasa natural de desempleo}

En el contexto del mercado laboral caracterizado por el proceso de calce descrito, la tasa de desempleo depende del flujo de salida hacia el desempleo de los individuos actualmente empleados, de la tasa de entrada al empleo de los cesantes y de los movimientos hacia dentro y fuera de la población inactiva. Si la economía se encontrara permanentemente en el "largo plazo" —o en una situación caracterizada por la ausencia de perturbaciones transitorias-, dichos flujos determinarían una tasa natural de desempleo, también conocida como desempleo friccional.

La legislación laboral sin duda puede impactar en el nivel de desempleo friccional en la medida en que afecte a las tasas de entrada y salida entre el empleo, el desempleo y la inactividad. Debido a que una mayor tasa natural implica una menor utilización de los factores productivos, países con desempleo más alto crecerán menos ${ }^{3}$.

\section{La productividad del trabajo}

La legislación puede afectar a la productividad del trabajo en la medida en que influya en el nivel de esfuerzo de los individuos. Por otro lado, si los cambios en la legislación afectan a las decisiones marginales de creación y destrucción de empleo - y por tanto, a la rotación del trabajo—- la productividad media de los trabajos existentes en cualquier momento del tiempo se

${ }^{3}$ Esta afirmación requiere de dos aclaraciones: primero, en un marco de crecimiento del tipo neoclásico con crecimiento de la productividad, cambios en la tasa natural llevan a cambios en el nivel de producto, pero no altera su tasa de crecimiento de largo plazo. Luego, aquí se está pensando en el crecimiento de trayectoria que implicaría una eventual baja en la tasa natural de desempleo. Segundo, esta afirmación lleva a cuestionamientos de la causalidad de las variables. Nuevamente, en un modelo del tipo descrito, sería el crecimiento del empleo el que lleva a un crecimiento del producto, y no al revés. 
verá afectada, ya que los trabajos creados y destruidos en el margen tienen productividades mayores y menores, respectivamente. Por último, la regulación influye en los incentivos para invertir en capital humano, variando el perfil del crecimiento de la productividad a lo largo de la vida del individuo.

\section{La tasa de inversión en capital}

Algunas teorías ${ }^{4}$ resaltan el rol de la especificidad de contratos incluida en la relación de producción entre el trabajo y el capital. Aumentos en el poder de negociación del factor trabajo llevarían, en el corto plazo, a una distribución de rentas a su favor como consecuencia de que el capital se encuentra "capturado" en una relación específica que le impide generar valor fuera del actual contrato. Sin embargo, en el largo plazo el capital exige su rendimiento alternativo "normal", lo que logra ajustando su oferta. Esto lleva a una sub-inversión en capital, menor acumulación de factores y, por tanto, a menor crecimiento.

\section{La participación en la población activa}

La regulación laboral en la mayoría de los países determina el tipo de contratos de trabajo que pueden celebrarse entre las partes interesadas, lo que afecta a la posibilidad de que ciertos grupos de la población puedan participar en el mercado. En particular, rigideces contractuales que regulan horarios y distribuciones de jornadas limitan la participación de mujeres y jóvenes - al menos dentro del marco de contratos legales_- y eso reduce el potencial productivo de la economía.

\subsection{Principales instituciones y su impacto en el mercado laboral}

Desde la década de los 80, diversos estudios han analizado el impacto de instituciones y regulaciones en los mercados laborales, motivados por el pobre desempeño en varias economías europeas que desde mediados de los 70 han experimentado aumentos persistentes en sus tasas de desempleo. Debido a que dicho fenómeno surge en un contexto de cambios en las regulaciones laborales - destinadas a proteger los salarios, puestos de trabajo y beneficios de desempleo, en presencia de agrupaciones sindicales de mayor poder-, los economistas culpan a dichas instituciones de haber rigidizado el mercado laboral, visión que parece razonable al contrastar con

${ }^{4}$ Ver Caballero y Hammour (1996) y McLeod y Malcomson (1993). 
el sistema anglo-americano de mercados menos regulados y de mejor desempeño en creación de empleos. Entre las principales instituciones y regulaciones mencionadas se encuentran:

\section{Protección al empleo: Costos de despido}

La mayoría de las economías incluyen en sus regulaciones los distintos tipos de restricciones al despido, como períodos de aviso, indemnizaciones por años de servicio y multas por despidos injustificados. Estas regulaciones afectan al funcionamiento del mercado laboral por varias razones: primero, afectan a la rotación laboral de la economía. Como expone Bertola (1990), la decisión de despido se toma cuando la productividad marginal de un trabajador cae por debajo de su salario. En presencia de los costos de despedir, sin embargo, la empresa ajusta su margen de despido, permitiendo que el salario supere al aporte de algunos trabajadores, ya que incurrir en el costo de la separación dejaría a la empresa en peor situación que si mantiene al trabajador de baja productividad por algún tiempo adicional, lo que tiende a reducir la productividad media de la empresa. Del mismo modo, a la hora de contratar la empresa descuenta los costos esperados futuros del eventual término de la relación laboral, lo que provoca distorsiones adicionales en la contratación y reduce la tasa de entrada al empleo. De esta manera, los costos de despido afectan a la rotación del trabajo y su productividad media, lo que a nivel agregado impacta en la tasa de crecimiento. Esta idea, esbozada por Blanchard y Portugal (1998), es confirmada empíricamente por Brown y Earle (2002) en un estudio para la transición económica en Rusia, quienes encontraron que regiones en que la transición se acompañó de una liberalización del mercado laboral exhibieron mayor rotación laboral e incrementos en la productividad.

Segundo, y muy relacionado con la idea anterior, los costos de despido pueden afectar a la tasa de desempleo natural de la economía, lo que dependerá en definitiva de si la disminución en la tasa de contrataciones es más pronunciada que la caída en las tasas de despido. Para Latinoamérica, un ejemplo es el trabajo de Kugler (2000), que analiza el impacto en rotación de la significativa reducción de los costos de despido en Colombia a comienzos de los noventa y encuentra que tanto la tasa de salida al desempleo como la tasa de entrada al empleo aumentan. El efecto neto es una disminución estimada en la tasa natural de desempleo entre 1,3\% y 1,7\%. Sin embargo, Blanchard y Portugal (1998), en un estudio comparativo entre Portugal y Estados Unidos, muestran que si bien la rotación total de empleos en un año es aproximadamente tres veces mayor en Estados Unidos, como resul- 
tado de los considerables niveles menores de costos de despido, la tasa de creación y destrucción neta de empleos es relativamente parecida entre ambos países, y por tanto exhiben una tasa de desempleo similar. Teórica y empíricamente, por tanto, existe una relación ambigua entre costos de despido y tasa de desempleo.

Tercero, si un alza en los costos de despido disminuye la probabilidad del mismo, la medida entrega incentivos para bajar el nivel de esfuerzo, afectando adicionalmente al nivel de productividad de los trabajadores, comportamiento que estaría respaldado por las teorías de "salarios de eficiencia” desarrolladas por Shapiro y Stiglitz (1984).

Por último, la menor rotación laboral causada por los costos de despido tiene su contraparte directa en el aumento de la duración del desempleo promedio. De acuerdo con el argumento de Blanchard y Katz (1997), dado que los trabajadores pierden habilidades laborales y eficiencia en la búsqueda de empleo con el paso del tiempo, muchos quedan "entrampados” en el ciclo vicioso de la desocupación, en detrimento de sus perspectivas futuras de trabajo. En efecto, Ham y Rea (1987) encuentran que en Canadá la duración del desempleo impacta negativamente en la probabilidad de salir del mismo.

\section{Protección al desempleo: Seguro de cesantía}

La excesiva generosidad de los beneficios referentes al desempleo se señala con frecuencia como la principal causa de los altos niveles de desempleo observados en Europa continental. En efecto, tal como expone Siebert (1997), los generosos beneficios otorgados, junto con el débil monitoreo del esfuerzo por parte de los beneficiarios, desincentivan la búsqueda de trabajo. Además de aumentar la tasa de desempleo, dichas políticas significan un peso enorme en los recursos fiscales, y terminan siendo insostenibles. Por otro lado, debido a que el mayor bienestar en el desempleo aumenta la agresividad negociadora de los sindicatos, el seguro introduce rigideces salariales adicionales que impactan en el nivel de empleo.

Por esta razón, mientras países como Irlanda, Inglaterra y Países Bajos han optado por reducir los beneficios del desempleo, otros los han mantenido constantes y han preferido intensificar el proceso de fiscalización de las actividades de búsqueda de empleo de los beneficiarios y las sanciones para quienes desechen trabajos "aceptables". Tal es el caso de Dinamarca, que continúa pagando el 90\% del último salario a los desempleados. Dado que esta opción es más costosa —Dinamarca gasta el 5\% del PIB 
en la administración del seguro-, otros países han optado por poner los incentivos directamente en los beneficiarios. Tal es el caso de Austria, y recientemente Chile, que financian el seguro con cuentas de capitalización individual.

Si bien los seguros de desempleo han sido fuertemente criticados en el pasado, la literatura económica más reciente apoya la existencia de este beneficio, basándose tanto en criterios de bienestar como de eficiencia, en la medida en que el seguro pase a reemplazar al ineficiente sistema de pago de indemnizaciones por años de servicio. En efecto, dado que los individuos valoran el perfil esperado de sus ingresos al igual que la estabilidad de los mismos, es razonable que deseen mantener un perfil de consumo estable durante el desempleo, lo cual muchas veces es imposible debido a las restricciones de acceso al mercado de crédito para ciertos individuos. En este sentido, el seguro de cesantía emula el contrato óptimo al que podrían llegar las empresas con sus trabajadores. Sin embargo, como se describe en Blanchard y Tirole (2005), dicho contrato es difícil de alcanzar directamente entre empresas y trabajadores porque es costoso monitorear los esfuerzos de búsqueda de los cesantes. Además, muchas empresas no son lo suficientemente grandes para ofrecer tal seguro, sobre todo dado el carácter sistémico de los despidos que tienden a concentrarse en recesiones. He aquí el rol del Estado como un intermediario, que brinda el servicio de administración, pago y monitoreo por medio de agencias — privadas o gubernamentales-.

Por lo tanto, el tema de fondo es cómo diseñar el seguro de desempleo para generar los incentivos adecuados. Entre los puntos a considerar se encuentran, primero, que los aportes se realicen en forma gradual para no agravar los problemas financieros de las empresas en ciclos recesivos. Esto genera además menos distorsiones en la decisión de despido, reduciendo los efectos negativos en rotación laboral y productividad. Segundo, el sistema de pagos y/o el monitoreo de los beneficiarios debe incentivar la búsqueda de empleos para evitar comprometer la solvencia estatal y distorsionar el proceso de calce. En efecto, Meyer (1990) encuentra que la probabilidad de salir del desempleo cae significativamente cuanto mayor sea la generosidad del beneficio, pero aumenta fuertemente la semana previa a que expire el seguro. Tercero, para que el sistema sea valorado por los trabajadores, los beneficios durante el desempleo deben depender de la mayor fuente de incertidumbre: su duración. La utilización de las indemnizaciones como método de protección ante el desempleo es por tanto doblemente ineficiente, desde el punto de vista de la decisión de la empresa y del bienestar de los trabajadores. 
Fijación de salarios indirecta: Atribuciones sindicales en la negociación colectiva

Hay bastante consenso en la teoría económica respecto al impacto del poder de negociación de los sindicatos en el equilibrio del mercado laboral. El principal argumento sostiene que en general a los sindicatos les interesa más el bienestar de sus miembros activos que las condiciones de la fuerza laboral en su totalidad. Por esta razón, muchas veces las demandas salariales no van en línea con las condiciones económicas imperantes, siendo común observar en países de alta afiliación sindical que en períodos de crisis sea el desempleo, en lugar del salario, el que termina ajustándose. Más aún, debido a que después de una recesión la cantidad de trabajadores afiliados generalmente ha disminuido debido a los despidos, el poder sindical aumenta, ya que las nuevas demandas salariales están destinadas a proteger a un menor número de trabajadores. Este tipo de argumentos se usa para explicar la persistencia del desempleo frente a shocks adversos (Lindbeck y Snower, 1987).

Por supuesto, el poder de negociación también depende de las características del mercado laboral. En presencia de alto desempleo, las negociaciones consideran el mayor costo que implica quedar desempleado —el llamado "efecto miedo"-, al tiempo que la empresa tiene más oferta externa para reemplazar a sus trabajadores —el "efecto amenaza"—. En general, sin embargo, la teoría predice que un mayor poder sindical debiera llevar a un mayor desempleo estructural y a un desempleo cíclico más persistente.

Otro efecto importante es sobre la inversión de capital de largo plazo. En efecto, como argumentan Caballero y Hammour (1998), el fuerte incremento en el poder sindical en Francia e Italia desde mediados de los setenta redundó, en el corto y mediano plazo, en menores rentas para el capital, niveles de desempleo levemente mayores y considerables alzas salariales. En el largo plazo, sin embargo, el capital se ajusta exigiendo su retorno competitivo, lo cual logra manteniendo a raya el poder sindical a través de un mayor nivel de desempleo. Esta historia explica perfectamente la evolución en estos países de la participación del capital —cae en un comienzo, y luego se recupera - y la del trabajo y los salarios — bajo desempleo y altos salarios hasta principio de los ochenta, luego alto desempleo, moderación salarial y una caída en la participación del trabajo en el producto-. Por supuesto, esto implica también una subinversión de capital, menor acumulación de factores y, por tanto, menor crecimiento económico. 
No obstante, no todos los países con alta afiliación sindical presentan mercados laborales rígidos y alto desempleo. En particular, Blanchard y Tirole (2005) cita las experiencias de los Países Bajos y Suecia, que exhiben alta afiliación sindical y han logrado limitar los aumentos salariales, reduciendo los altos niveles de desempleo. Esta idea fue originalmente estudiada por Calmfors y Driffill (1988), quienes plantean que lo importante no es el nivel de afiliación sindical, sino la coordinación entre los sindicatos de distintos sectores. Así, en países donde la coordinación es fluida, las demandas salariales consideran los efectos en la economía agregada, evitando caer en excesos.

\section{Fijación de salarios directa: Salarios mínimos}

La fijación de salarios mínimos es una práctica tradicional en la mayoría de los países del mundo con el objetivo de combatir la pobreza, y fue instaurada para asegurar que los trabajadores menos calificados reciban una compensación “justa” por su esfuerzo. Sin embargo, la teoría económica tradicional considera que tal política puede tener importantes implicancias en los niveles de desempleo, ya que individuos de baja calificación que contribuyen menos que el salario mínimo se vuelven "inempleables".

Sin embargo, algunos economistas apoyan la fijación de salarios mínimos bajo la premisa de que existen imperfecciones en los mercados laborales, como problemas informacionales y costos de transacción al cambiar de empleo, que otorgan a las empresas un poder de negociación que les permite pagar un salario menor que el del equilibrio competitivo. Luego, una fijación de salarios mínimos menores o iguales al equilibrio competitivo, pero mayores que los vigentes en el mercado imperfecto, tendría efectos inambiguos en reducir la pobreza, aumentando el salario sin afectar al empleo. El efecto en el bienestar de los trabajadores menos calificados depende entonces de cuál enfoque es el más apropiado para caracterizar la situación de dicho segmento del mercado laboral (Bravo y Vial, 1997), pregunta que debe responderse por la evidencia empírica ${ }^{5}$.

La evidencia internacional reciente encuentra en diversos estudios que el efecto de los salarios mínimos en el empleo no es significativo ${ }^{6}$. Para Chile existen diversos estudios empíricos que abordan el tema del efecto en salario mínimo. La conclusión que prevalece, a pesar de ciertas excepcio-

${ }^{5}$ Un elemento adicional a considerar es el eventual impacto que un mayor salario mínimo tiene sobre la tasa de deserción escolar, lo que comprometería los ingresos futuros. Este punto es abordado por Paredes y Sanhueza (1996).

${ }^{6}$ Ver Card y Krueger (1995) para una discusión al respecto. 
nes $^{7}$, es que el salario mínimo tiene un impacto negativo en el segmento de trabajadores más jóvenes y de menor calificación, tal como encuentran Bravo y Contreras (2001) y Cowan et al. (2003). La discusión está, por lo tanto, en qué tan restrictivo es el salario mínimo en un determinado país.

\section{Regulación de contratos}

La regulación laboral condiciona el tipo de contrato que pueden acordar las partes. El contrato más utilizado es el de plazo indefinido, sobre el cual se aplican las normas estándares de terminación de contrato y cotizaciones previsionales y de cesantía. Este tipo de contrato, sin embargo, presenta serios problemas para diversos segmentos de la población, especialmente en jóvenes y mujeres, que se ven imposibilitados de cumplir con la jornada completa, o presentan un riesgo muy grande para las empresas que no están dispuestas a pagar los costos de terminación de contratos de alta incertidumbre. En este contexto, muchos países han aplicado contratos de mayor flexibilidad, entre los cuales se encuentran los contratos a plazo fijo, contratos temporales y empresas externas que los intermedian, jornadas de tiempo parcial y la posibilidad de variar las horas trabajadas dentro de períodos determinados, mientras otros aceptan períodos de prueba y/o normas especiales para contratos de aprendizaje.

Existe amplia literatura empírica que evalúa los impactos de dichos contratos en los niveles de empleo, salarios y productividad ${ }^{8}$. Jimeno y Rodríguez-Palenzuela (2002) encuentran que una regulación menos estricta de contratos a plazo fijo aumenta el empleo juvenil. Para España, Kugler et al. (2003) concluyen que la reducción en los costos de despido de los más jóvenes aumentó su nivel de empleo, mientras que Blanchard y Landier (2002) revelan que la introducción de contratos a plazo fijo en Francia aumentó significativamente la rotación laboral de los más jóvenes, aunque sin un impacto claro en el empleo, resultado similar al de Hopenhayn (2001) para Argentina.

En un estudio acerca del impacto de contratos con flexibilidad de horario, Kim y Campagna (1981) muestran que la opción del trabajador y la empresa de reorganizar las jornadas aumenta la productividad y reduce la tasa de ausentismo. Respecto al desarrollo de empresas que intermedian trabajo temporal, Nunziata y Staffolani (2001) encuentran en un estudio para nueve países de la OECD un impacto positivo de empleo en trabajos tempo-

${ }^{7}$ Ver Bravo y Vial (1997), para una discusión de diversos trabajos.

${ }^{8}$ Ver Dolado et al. (2005) para una discusión teórica y empírica de diversos trabajos relacionados. 
rales y a nivel agregado, pero sólo durante ciclos recesivos. Respecto al impacto de los contratos de jornada parcial, un estudio de la OECD (2003a) estima que es, lejos, el factor más importante para explicar diferencias entre países en la participación laboral femenina, seguido del sistema de subsidios al cuidado infantil.

\subsection{Evidencia directa de instituciones y crecimiento}

Si bien la sección anterior alude a diversos estudios empíricos para evaluar el impacto de distintas instituciones en las principales dimensiones del mercado laboral, para el propósito del presente trabajo es necesario revisar la literatura que estima directamente la relación entre regulación laboral y crecimiento. En particular, existe una línea de investigación que relaciona crecimiento con medidas extensas de institucionalidad laboral en cada país, más que de regulaciones aisladas.

Besley y Burges (2004) estudian el impacto de una serie de reformas laborales aplicadas de manera diferenciada a la industria manufacturera entre los estados de India. Descubren que la regulación pro trabajador tiene un efecto negativo y significativo en la producción industrial —además de estar más asociada con pobreza en zonas urbanas-, resultado que es similar al encontrado anteriormente por Holmes (1998) para la evidencia interestatal en Estados Unidos. Botero et al. (2003) clasifican regulaciones laborales en 85 países y muestran que un marco más estricto está asociado con un mayor tamaño de la economía informal, mayor desempleo y menor participación. Calderón y Chong (2005) estiman el impacto en la tasa de crecimiento de diversas regulaciones, utilizando las bases de datos de Forteza y Rama (2002) y Botero et al. (2003) de distintos aspectos de la regulación laboral. El trabajo distingue medidas de regulación "en el papel” — tales como el número de regulaciones laborales de la OIT ratificadas por cada país - y regulaciones “en la práctica” — como el salario mínimo, la afiliación sindical, las imposiciones al seguro social y el empleo público-. Encuentran que una disminución en la regulación de ambos tipos de medidas aumentaría las tasas de crecimiento para economías en desarrollo, mientras que sólo una desregulación “en la práctica” sería útil para los países industrializados. Los efectos no serían menores: por ejemplo, si Argentina (el país de mayor regulación del primer tipo en Latinoamérica) bajara su regulación al nivel del país promedio, su crecimiento aumentaría en $0,8 \%$ cada año. El efecto es similar si se reducen las regulaciones del segundo tipo, con un mayor impacto en crecimiento de economías desarrolladas 
—hasta $2 \%$ cada año- . Sin embargo, los autores reconocen que dicho crecimiento sólo es alcanzable con grandes transformaciones de la institucionalidad legal y que políticas marginales aisladas no tendrían —ni han tenido — un impacto significativo.

\section{Diagnóstico del mercado laboral chileno}

\subsection{Características estructurales del mercado laboral}

a) Aspectos estructurales de largo plazo

La Figura $\mathrm{N}^{\circ} 1$ muestra la evolución del desempleo y de los salarios reales en Chile durante el período 1980-2004. Entre los aspectos estructurales interesantes de destacar, el salario real ha crecido en torno al 2,3\% anual promedio, bastante en línea con el crecimiento de la productividad, en torno al 2\%. El desempleo registra un promedio de 9,6\% en el período considera-

FIGURA N ${ }^{\circ}$ 1: $\quad$ DESEMPLEO, SALARIOS Y PARTICIPACIÓN, 1980-2004

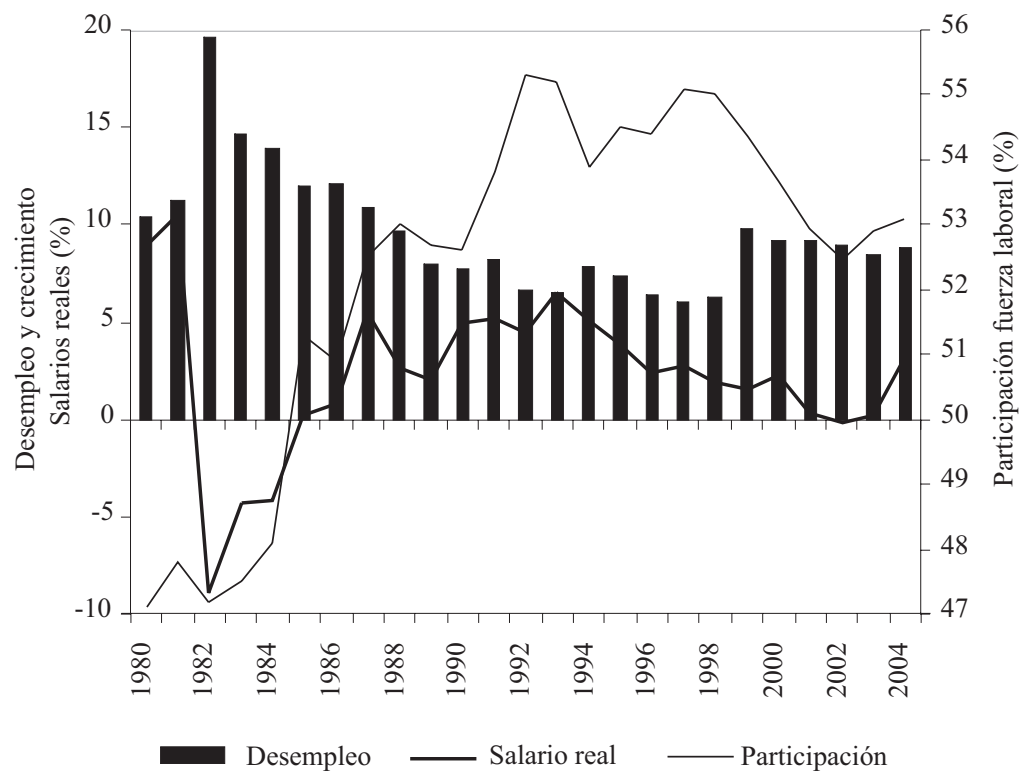

Fuente: INE. 
do, mientras que en la "época dorada" de crecimiento entre 1987-1997 fue tan sólo de 7,7\%. La participación de la fuerza laboral ha estado en torno al $52 \%$ de la población en edad de trabajar (definida sobre los 15 años), la cual correspondería a un 32\% de participación para las mujeres y un 74\% para los hombres.

El Cuadro $\mathrm{N}^{\circ} 1$ muestra algunas variables del mercado laboral para un amplio grupo de economías ${ }^{9}$. Destaca el alto desempleo en Chile, superior incluso al promedio de Europa continental. Más preocupante aún es la poca participación de las mujeres y la de los jóvenes, cuyos niveles son la primera menor y la sexta menor, respectivamente, entre los 38 países considerados.

\section{b) Aspectos cíclicos y evolución reciente}

A partir de la crisis asiática, la tasa de desempleo en Chile subió a niveles en torno al $10 \%$, mostrando una fuerte persistencia hasta fines de 2004, algo por debajo del 9\%. Poco después del comienzo de la crisis, durante 1999, el Congreso comenzó a debatir un conjunto de iniciativas legales que modificaban diversos aspectos de la legislación laboral vigente, los cuales fueron aprobados y comenzaron a operar en 2001. Entre las reformas destacan el aumento en las multas a despidos injustificados, mayores atribuciones sindicales para la negociación colectiva y la flexibilización de horarios y jornadas parciales, así como la aprobación de un seguro de desempleo poco tiempo después. Este paquete de reformas causó polémica en distintos sectores y un rechazo entre muchos economistas que lo consideraron un paso hacia el modelo equivocado de regulación laboral, más en línea con la institucionalidad vigente en países europeos que han tenido malos desempeños en el mercado del trabajo.

La preocupación planteada no carece de fundamentos. Por un lado, debido al carácter procíclico de la fuerza laboral (participación), muchos desempleados salen de la cesantía hacia la inactividad durante períodos de recesión, lo que reduce la tasa media de desempleo subestimando el verdadero problema de desocupación. Por otra parte, el fuerte aumento de la informalidad muestra que muchos de los empleos creados han sido al margen de toda regulación laboral (Figura $\mathrm{N}^{\circ}$ 2).

${ }^{9}$ Los datos de participación corresponden al último año para el cual la OIT tiene información (entre 2000 y 2001), mientras que el dato de desempleo corresponde al promedio para el período 1980-2000. 
CUADRO N N $^{\circ}$ : DESEMPLEO Y PARTICIPACIÓN LABORAL, PAÍSES SELECCIONADOS (\%)

\begin{tabular}{|c|c|c|c|c|c|c|c|}
\hline \multirow[t]{2}{*}{ País } & \multirow{2}{*}{$\begin{array}{r}\text { Desempleo } \\
1980-2000\end{array}$} & \multicolumn{3}{|c|}{ Participación mayores de 15 años } & \multicolumn{3}{|c|}{ Participación 15-24 años } \\
\hline & & Total & Hombres & Mujeres & Total & Hombres & Mujeres \\
\hline Austria & 4,7 & 59,0 & 69,4 & 49,5 & 58,4 & 62,6 & 54,2 \\
\hline Bélgica & 11,4 & 51,9 & 61,2 & 43,2 & 32,9 & 35,5 & 30,1 \\
\hline Dinamarca & 9,3 & 65,8 & 71,8 & 60,0 & 73,3 & 76,7 & 70,1 \\
\hline Finlandia & 11,0 & 66,4 & 69,5 & 63,3 & 50,6 & 50,4 & 50,8 \\
\hline Francia & 10,2 & 66,9 & 77,2 & 57,4 & 29,5 & 32,7 & 26,2 \\
\hline Alemania & 8,4 & 58,4 & 68,9 & 48,7 & 52,5 & 57,1 & 47,6 \\
\hline Italia & 10,6 & 48,3 & 62,2 & 35,3 & 39,6 & 45,1 & 34,0 \\
\hline Luxemburgo & 2,2 & 52,4 & 64,3 & 40,7 & 34,0 & 36,0 & 31,9 \\
\hline Países Bajos & 7,8 & 64,8 & 80,5 & 48,7 & 67,7 & 67,4 & 68,0 \\
\hline Noruega & 3,8 & 73,5 & 78,0 & 68,9 & 64,7 & 67,5 & 61,8 \\
\hline Portugal & 6,6 & 61,0 & 70,2 & 52,7 & 47,3 & 51,2 & 43,4 \\
\hline España & 18,9 & 50,6 & 63,9 & 38,4 & 47,4 & 52,7 & 41,8 \\
\hline Suecia & 4,4 & 70,0 & 73,4 & 66,6 & 51,1 & 52,6 & 49,5 \\
\hline Suiza & 2,2 & 67,4 & 77,8 & 57,6 & 68,3 & 70,5 & 66,0 \\
\hline Europa continental & 8,0 & 61,2 & 70,6 & 52,2 & 51,2 & 54,1 & 48,2 \\
\hline Canadá & 9,5 & 65,9 & 72,5 & 59,5 & 64,4 & 65,9 & 62,9 \\
\hline Islandia & 2,4 & 75,9 & 81,1 & 70,8 & 68,1 & 66,2 & 70,1 \\
\hline Irlanda & 14,4 & 57,8 & 70,1 & 46,0 & 50,7 & 54,3 & 46,9 \\
\hline Japón & 2,6 & 62,4 & 76,4 & 49,3 & 47,0 & 47,4 & 46,6 \\
\hline Inglaterra & 8,8 & 62,9 & 71,6 & 54,5 & 69,2 & 73,2 & 65,0 \\
\hline Estados Unidos & 6,6 & 67,2 & 74,7 & 60,2 & 65,9 & 68,6 & 63,2 \\
\hline Australia & 8,2 & 62,5 & 72,0 & 53,2 & 68,4 & 70,8 & 65,9 \\
\hline Nueva Zelanda & 6,1 & 65,3 & 73,6 & 57,5 & 63,3 & 66,9 & 59,6 \\
\hline Otros OECD & 7,3 & 65,0 & 74,0 & 56,4 & 62,1 & 64,2 & 60,0 \\
\hline Polonia & 12,8 & 56,7 & 64,5 & 49,6 & 34,7 & 37,9 & 31,5 \\
\hline Rep. Checa & 5,5 & 60,4 & 69,8 & 51,6 & 46,1 & 51,3 & 40,6 \\
\hline Hungría & 9,1 & 53,1 & 61,4 & 45,4 & 40,7 & 46,2 & 35,0 \\
\hline Eslovaquia & 13,9 & 59,6 & 67,7 & 52,2 & 45,6 & 48,6 & 42,6 \\
\hline Europa oriental & 10,3 & 57,5 & 65,9 & 49,7 & 41,8 & 46,0 & 37,4 \\
\hline Hong Kong, China & 2,7 & 62,0 & 75,5 & 48,5 & 49,3 & 49,5 & 49,1 \\
\hline Corea & 3,6 & 60,5 & 74,4 & 47,4 & 31,3 & 26,5 & 35,4 \\
\hline Tailandia & 2,9 & 72,2 & 80,3 & 64,2 & 53,4 & 57,9 & 48,6 \\
\hline Indonesia & 3,1 & 67,9 & 84,6 & 51,5 & 54,0 & 65,0 & 43,0 \\
\hline Singapur & 3,2 & 63,9 & 77,5 & 51,3 & 44,2 & 42,5 & 45,8 \\
\hline Asia-Pacífico & 3,1 & 65,3 & 78,5 & 52,6 & 46,4 & 48,3 & 44,4 \\
\hline Argentina & 8,7 & 58,2 & 76,2 & 41,3 & 52,3 & 63,1 & 41,2 \\
\hline Brasil & 4,8 & 66,9 & 82,0 & 52,8 & 63,0 & 75,1 & 50,7 \\
\hline Chile & 9,5 & 55,0 & 74,4 & 36,5 & 35,2 & 44,6 & 25,1 \\
\hline Colombia & 11,8 & 67,7 & 79,8 & 57,7 & 53,8 & 58,6 & 49,7 \\
\hline Perú & 5,9 & 68,2 & 79,4 & 58,1 & 57,0 & 61,7 & 52,7 \\
\hline Venezuela & 9,7 & 60,9 & 81,4 & 40,4 & 46,1 & 62,7 & 28,9 \\
\hline México & 4,0 & 60,0 & 83,8 & 38,5 & 52,5 & 69,8 & 36,1 \\
\hline Latinoamérica & 7,8 & 62,4 & 79,6 & 46,5 & 51,4 & 62,2 & 40,6 \\
\hline
\end{tabular}

Fuente: OIT, Rama y Artecona (2002) y FMI. 
FIGURA N ${ }^{\circ}$ 2: $\quad$ PARTICIPACIÓN E INFORMALIDAD

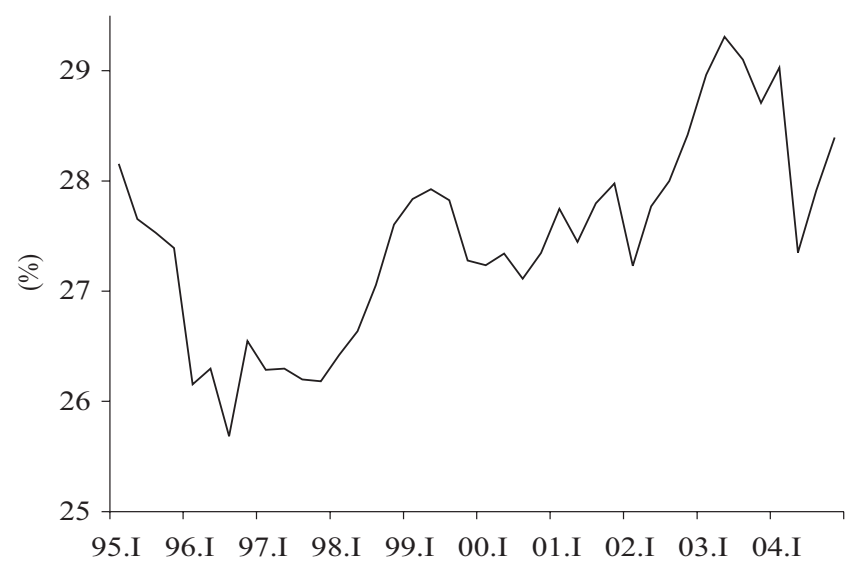

Empleo informal

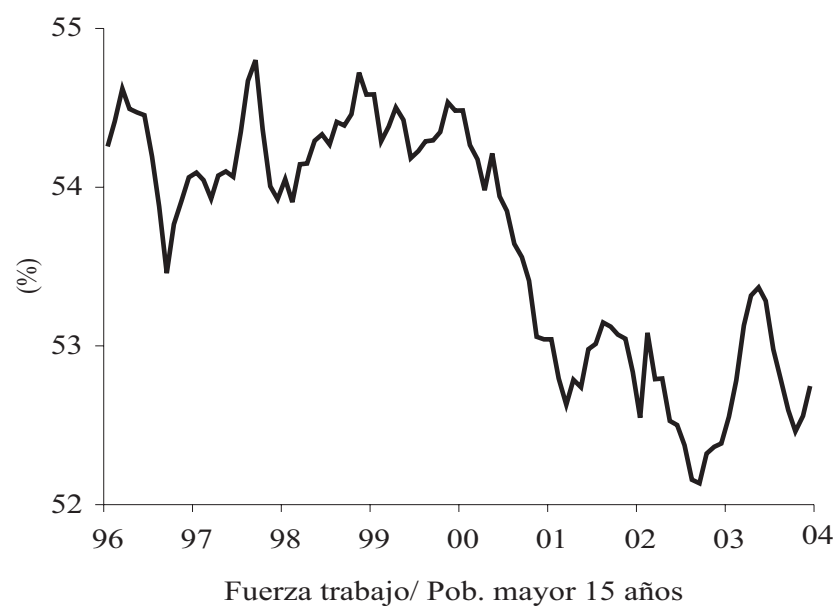

Fuente: INE. Informalidad definida como trabajadores por cuenta propia + familiar no remunerado.

Sin embargo, una visión alternativa interpreta la evolución del desempleo en los últimos años como un fenómeno cíclico que responde a la desaceleración del PIB causada por la crisis asiática, a la que siguieron la caída de los términos de intercambio, el 11 de septiembre, la turbulencia en Argentina y Brasil y la crisis en Iraq, entre otros. La persistencia del desempleo sería un reflejo de varios shocks, no de un mercado laboral intrínseca- 
mente rígido, y en este caso el actual marco legal está lejos de los estándares europeos al diferenciar entre regulaciones "en el papel” y sus repercusiones “en la práctica” en un país con aplicación más débil de las leyes.

En efecto, Cowan et al. (2003) estiman que la caída del empleo puede explicarse por shocks negativos de demanda, debido a que la alta rigidez de salarios provoca que las caídas en la demanda por trabajo se reflejen en mayor desempleo. Detrás de dicha rigidez estarían la negociación a dos años con indexación a la inflación pasada ${ }^{10}$ y el fuerte aumento del salario mínimo por tres años consecutivos decretado en $1998^{11}$, que afectaría a los salarios del sector público y privado. Más aún, estiman efectos negativos en empleo de los más jóvenes y menos calificados. En efecto, con relación al salario medio, el salario mínimo en Chile es alto incluso para los estándares de economías industrializadas (Figura $\mathrm{N}^{\circ} 3$ ).

Ante estas visiones, es útil revisar el estado actual de la legislación laboral chilena, así como la posición en el contexto internacional, ejercicio necesario a la hora de buscar focos de mejoría para perfeccionar el funcionamiento del mercado del trabajo e impulsar el crecimiento económico.

FIGURA N ${ }^{\circ}$ 3: $\quad$ SALARIO MÍNIMO RELATIVO AL SALARIO MEDIO: COMPARACIÓN INTERNACIONAL

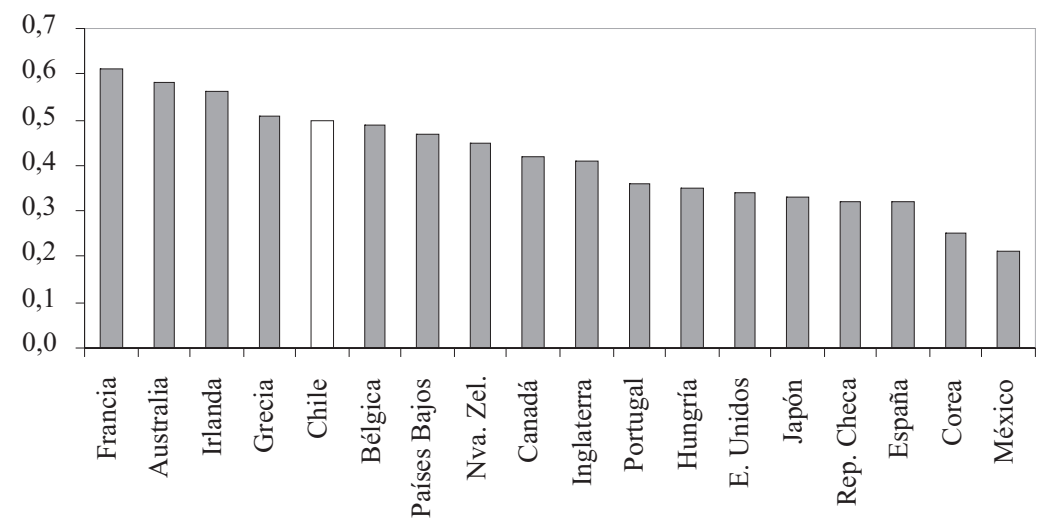

Fuente: OECD: Economic Surveys, Chile 2003.

10 Tal como lo señala Jadresic (1997), esto hace que la respuesta del empleo sea más persistente a los shocks externos.

${ }^{11}$ En montos de 12,7\%, 12,4\% y 10,5\%, respectivamente. 


\subsection{Instituciones laborales vigentes}

Indemnizaciones por años de servicio (IAS)

Al igual que en la mayoría de los países, la legislación chilena da derecho al trabajador despedido a recibir una IAS correspondiente a un mes por año de servicio prestado con un tope legal de 11 años, dando la posibilidad de negociar pagos a todo evento a partir del séptimo año. Las causas aceptadas para el despido son necesidades de la empresa por condiciones económicas o reestructuración de procesos, además de ausentismo injustificado, robos e injurias, entre otras. No obstante, si el trabajador alega despido injustificado, el fallo a su favor de los tribunales del trabajo implica incrementar el pago de indemnizaciones entre 30\% y 100\% sobre el monto original. El pago de las indemnizaciones debe realizase a más tardar 60 días después de terminado el contrato en un solo pago, con perjuicio de multa, de no cumplir esta disposición, de $150 \%$ del monto original ${ }^{12}$.

Seguro de desempleo

La implementación del seguro de desempleo en Chile es sin duda el cambio más trascendente de la reciente reforma. Éste considera el pago de beneficios proporcionales al sueldo recibido anteriormente, con un máximo de 5 meses, durante los cuales el importe recibido disminuye gradualmente. El financiamiento proviene de cuentas de capitalización individual, las que se financian con imposiciones mensuales proporcionales al sueldo bruto en parte por los trabajadores $(0,6 \%)$, en parte por las empresas $(1,6 \%)$, y para trabajadores de bajos ingresos, en parte también por el fondo solidario creado con un aporte de la empresa del sueldo de cada trabajador (0,8\%). El uso del beneficio está restringido a un máximo de 2 veces en 5 años.

\section{Atribuciones sindicales}

El contrato colectivo se celebra para establecer condiciones comunes de trabajo y de remuneraciones. Se requiere un mínimo de 25 trabajadores (que representen al menos el $10 \%$ del total) para empresas de más de 50 , y al menos 8 para empresas menores.

${ }^{12}$ Entre las cláusulas contempladas en la nueva reforma se encuentran el incremento de las multas por despido injustificado del rango entre $20 \%$ y $50 \%$, el nuevo castigo por pago fuera de plazo, y que la falta de adecuación laboral o técnica del trabajador ya no es aceptada como una causal justificada de despido. 
En la negociación colectiva existen una serie de prácticas consideradas antisindicales por parte del empleador, incluyendo negarse a proporcionar información financiera de la empresa y la planilla de costos laborales, que son sancionadas con multas de 10 a 150 unidades tributarias mensuales ${ }^{13}$. Para los directivos sindicales existen una serie de normativas que permiten dedicar parte de la jornada laboral a actividades del sindicato —sujeto a remuneración—, así como restricciones a su despido.

La negociación colectiva se inicia con una propuesta que debe responder la empresa dentro de 15 días, por la cual la comisión negociadora puede exigir al menos la suscripción de un nuevo contrato con iguales estipulaciones al vigente. Si la mayoría de los trabajadores no aceptan la última oferta del empleador, podrán declarar la huelga. El empleador podrá contratar otros trabajadores a partir del primer día, pero deberá pagar un bono de reemplazo de $4 \mathrm{UF}$ al sindicato por cada trabajador contratado ${ }^{14}$.

\section{Salario mínimo}

El salario mínimo es revisado en junio de cada año. En la actualidad asciende a \$ 127.500 mensuales, cercano a la mitad del valor del salario medio. Se fija a veces por un año, y en ocasiones por plazos mayores. Su valor es propuesto por el gobierno -influenciado en mayor o menor medida por presiones sindicales y empresariales - y finalmente debe aprobarlo el Congreso. La ley permite un salario mínimo diferenciado para menores de 18 y mayores de 65 años, equivalente al 75\% del salario mínimo base.

\section{Tipo de contratos}

El contrato estándar en Chile es el de plazo indefinido, sobre el cual aplican todos los beneficios y cargos de previsión social, así como las compensaciones por despido. En el caso de los contratos a plazo fijo, la ley limita su duración a un plazo de un año, cuya renovación por segunda vez implica presunción de contrato indefinido. Un tipo especial de contrato se refiere a la contratación de aprendices, que están exentos del salario míni$\mathrm{mo}^{15}$. Una innovación de la reciente reforma permite descontar del pago de indemnizaciones los gastos en capacitación (con un tope de un mes), con el fin de incentivar la inversión en capital humano. Otra importante modificación es la posibilidad de pactar contratos de tiempo parcial de menos de 30 horas semanales.

131 UTM de agosto 2004 equivale a \$ 30.000 aprox.

14 Normativa incluida en la nueva reforma.

15 Aplicable sólo a menores de 21 con un máximo de 2 años y un límite del $10 \%$ de la planilla total. 


\section{3. ¿Qué tan rígido es el mercado laboral chileno?}

La literatura económica ha recurrido por lo común a dos enfoques para comparar rigideces laborales entre los distintos países. Por un lado, algunos estudios construyen indicadores "legislativos", que miden y ponderan de alguna manera las distintas normativas legales imperantes en cada país. No son muchos, sin embargo, los estudios que incluyen a países latinoamericanos en la muestra considerada. Tal es el caso de Heckmann y Pagés (2000), que construyen un indicador de costos de despido que incluye el costo estimado incurrido en los meses de aviso, la compensación directa por despido, y gastos esperados por juicios y multas. Según este indicador, la rigidez en Latinoamérica sería considerablemente mayor que la de países desarrollados y Chile está en línea con el promedio regional, en niveles comparables con España.

Rama y Artecona (2002) construyen indicadores legales y de desempeño laboral para 121 países desde mediados de los años cuarenta hasta los noventa, en dimensiones como costos de despido, condiciones laborales y la cantidad de códigos de la OIT acogidos en la regulación. El indicador de rigidez promedio que calculan muestra resultados similares para Latinoamérica, aunque Chile se encuentra por debajo del promedio regional, con niveles algo superiores a la media de países desarrollados ${ }^{16}$. Otro ranking publicado por la OECD (2003b), construido en forma similar pero que incluye los cambios de la reforma reciente, ubica a Chile en el rango medio de los países considerados, destacando nuevamente los altos costos de despido.

Por otra parte, existe una línea de investigación que evalúa la rigidez de los mercados laborales de acuerdo con su desempeño frente a shocks estructurales, prescindiendo de indicadores “legales”, siguiendo la idea de que la regulación importa en la medida en que las instituciones de los distintos países las lleven a la práctica, lo que depende del grado de desarrollo. Albagli et al. (2005) rankean a Chile en una muestra constituida por 18 países desarrollados y en transición con un indicador basado en la persistencia ${ }^{17}$ del desempleo después que la economía se ve afectada por algún shock. Los resultados indican que Chile presenta uno de los mercados más

16 Cabe mencionar, no obstante, que ninguno de estos rankings considera la legislación chilena posterior a la reforma de 2001, por lo que los índices actualizados debieran mostrar mayor rigidez.

17 Concretamente, se calcula en base a la vida media de los shocks, o cuánto se demora el desempleo en volver la mitad del camino desde su punto de mayor desvío hacia su nivel natural. Pese a usar metodologías distintas, la estimación de 4,6 trimestres está en línea con otras estimaciones similares. Caballero et al. (2005) y Martínez et al. (2001) la estiman en 2,5 trimestres, mientras que Cowan et al. (2003) en 5,6 trimestres. 
flexibles, sugiriendo que, con excepción de Colombia, que confirma la rigidez estimada de acuerdo a medidas legales, la rigidez verdadera o "en la práctica” de Chile (y México) sería sustancialmente menor. Los principales indicadores de rigidez de estos estudios, junto a medidas de desempleo, se muestran en el Cuadro $\mathrm{N}^{\circ} 2$.

CUADRO N 2: $\quad$ COMPARACIÓN DE INDICADORES DE RIGIDEZ

\begin{tabular}{|c|c|c|c|c|}
\hline País & $\mathrm{HP}^{\mathrm{a}}$ & $\mathrm{RA}^{\mathrm{b}}$ & $\mathrm{OECD}^{\mathrm{c}}$ & $\mathrm{AGR}^{\mathrm{d}}$ \\
\hline Alemania & 9,49 & 51,25 & 2,60 & 10,75 \\
\hline Austria & 14,86 & 29,50 & 2,40 & 10,50 \\
\hline Bélgica & 14,40 & 48,25 & 2,40 & 9,38 \\
\hline Francia & 9,52 & 50,00 & 2,80 & 9,00 \\
\hline Dinamarca & N.A. & 25,50 & 1,50 & 9,67 \\
\hline España & 26,30 & 69,75 & 3,10 & 11,38 \\
\hline Países Bajos & N.A. & 54,25 & 2,20 & 7,33 \\
\hline Italia & N.A. & 59,25 & 3,40 & 9,58 \\
\hline Portugal & 34,72 & 79,00 & 3,70 & N.A. \\
\hline Suecia & N.A. & 42,00 & 2,60 & 12,13 \\
\hline Europa continental & 18,22 & 50,83 & 2,67 & 9,97 \\
\hline Estados Unidos & 0,00 & 22,25 & 0,70 & 5,75 \\
\hline Canadá & 4,60 & 33,75 & 1,10 & 6,75 \\
\hline Australia & 3,69 & 35,75 & 1,20 & 7,50 \\
\hline Corea & N.A. & 51,00 & 2,50 & 4,33 \\
\hline Nueva Zelanda & 1,84 & 32,00 & 0,90 & N.A. \\
\hline Inglaterra & 12,10 & 28,00 & 0,90 & 7,38 \\
\hline Otros OECD & 4,45 & 33,79 & 1,22 & 6,34 \\
\hline Argentina & 24,80 & 66,00 & N.A. & N.A. \\
\hline Bolivia & 39,63 & 66,00 & N.A. & N.A. \\
\hline Brasil & 14,87 & 78,25 & N.A. & N.A. \\
\hline Chile & 28,16 & 50,00 & 1,80 & 4,63 \\
\hline Colombia & 29,10 & 59,25 & N.A. & 22,75 \\
\hline México & 26,05 & 77,25 & 2,60 & 5,50 \\
\hline Perú & 31,63 & 73,50 & N.A. & N.A. \\
\hline Uruguay & 18,59 & 39,00 & N.A. & N.A. \\
\hline Venezuela & 24,62 & 75,25 & N.A. & N.A. \\
\hline Latinoamérica & 26,38 & 64,94 & 2,20 & 10,96 \\
\hline
\end{tabular}

a Fuente: Heckman y Pagés (2000). Datos corresponden a fines de los 90.

${ }^{\mathrm{b}}$ Fuente: Rama y Artecona (2002). Datos de promedios quinquenales, desde los 40 hasta los 90 .

c Fuente: OECD Employment Outlook, 1999. Incluye condiciones hasta 1998 para OECD.

d Fuente: Albagli et al. (2005). Construido a partir de datos trimestrales, entre 1980-2002. 
En resumen, Chile se encuentra alrededor de la media en los rankings que evalúan varias dimensiones del mercado laboral (RA, OECD), destacando los altos costos de despido por el pago de indemnizaciones, costos judiciales y multas (HP). En términos de desempeño, sin embargo, la persistencia del desempleo frente a shocks es baja en comparación con los países desarrollados (AGR). Entre los problemas estructurales más graves se encuentra la baja participación laboral de las mujeres y los jóvenes, en que el escaso uso de contratos de tiempo parcial, la rigidez de jornada y los contratos a plazo fijo, así como el alto salario mínimo en relación con el medio, parecen ser los principales responsables.

\section{Propuestas y desafíos pendientes}

En esta sección se discuten propuestas para aumentar la flexibilidad del mercado laboral chileno y enfrentar sus principales problemas estructurales de manera de aumentar los niveles de participación y empleo, la productividad del trabajo y el crecimiento económico en un marco de protección al trabajo que sea eficiente desde el punto de vista macroeconómico. El orden de las propuestas no es arbitrario, comenzando con las de mayor prioridad de acuerdo con lo que se desprende del análisis teórico y empírico presentado anteriormente, a la luz de la situación propia del país.

\subsection{Costos de despido: Seguro de desempleo en lugar de IAS}

El diseño del seguro de cesantía aplicado en Chile cumple varias de las características deseables de un sistema de protección eficiente en un país en vías de desarrollo. Sin embargo, debido a que su implementación es independiente del sistema IAS, la medida significa un costo laboral adicional para la empresa. Debido a que, respecto a estándares internacionales, los costos esperados de despido (y por ende de contratación) se encuentran en rangos bastante elevados, la primera propuesta es reducir las IAS privilegiando el sistema del seguro de cesantía. El objetivo es que, independientemente del monto de recursos involucrados en la decisión de separación laboral, éstos dejen de ser percibidos como un costo por parte del empleador, así como un beneficio por parte del empleado, que se genera por la situación de despido (o renuncia) en sí, con las consecuentes repercusiones en rotación laboral y eficiencia discutidas anteriormente. En efecto, si se optara por la alternativa de que la totalidad del monto pagado de una sola 
vez por la empresa al momento de la separación fuera alternativamente cotizada mensualmente en el fondo de cesantía, la decisión de separación por parte de la empresa no consideraría dicho costo, así como para el empleado no implicaría un beneficio en el sentido de que cualquier pago es financiado por su propia cuenta de cesantía.

La propuesta no pretende determinar si dicho monto (el porcentaje cotizado en caso de reformarse) es hoy excesivo o insuficiente, lo que depende al final de preferencias sobre las cuales es arriesgado hacer juicios normativos. En principio, un mayor pago (bajo cualquier esquema) por término de la relación laboral implica también menores salarios en equilibrio, tal como una prima por aseguramiento será más cara mientras más generosa sea la indemnización en caso de producirse el evento adverso. La idea es simplemente que, dado el monto de recursos involucrados hoy en proteger el bienestar del trabajador en sus episodios de cesantía, las IAS son una manera ineficiente de hacerlo, al generar incentivos perversos en detrimento de la productividad y el crecimiento ${ }^{18}$.

Medidas complementarias para impulsar el funcionamiento del seguro incluyen perfeccionar la actividad de las oficinas municipales de información de empleo en coordinación con el seguro de cesantía, en lo que la literatura denomina medidas "activas” de protección laboral dirigidas a disminuir la duración del desempleo ${ }^{19}$.

4.2. Participación laboral de mujeres y jóvenes: Flexibilidad de contratos, costos de maternidad y salario mínimo

Sin duda, uno de los principales problemas del mercado laboral chileno es la baja participación de las mujeres y de los segmentos más jóvenes de la población. Si bien esto puede estar relacionado con factores culturales ${ }^{20}$, la rigidez implícita en los contratos vigentes puede tener efectos muy importantes en la baja tasa de participación y limitar la capacidad productiva de la economía.

${ }^{18}$ Pese a que la ley permite descontar de las indemnizaciones los aportes al seguro de cesantía, las cotizaciones actuales son relativamente bajas respecto al monto de las indemnizaciones, por lo que las distorsiones mencionadas continuarán en pie hasta que no se tomen medidas adicionales.

${ }^{19}$ Evaluaciones de estos programas en otros países entregan resultados mixtos. Calmfors et al. (2002) encuentran para Suecia un efecto nulo en la probabilidad de encontrar empleo, mientras que Blundell y Costas (2002) evalúan positivamente políticas similares en Inglaterra. Sería clave considerar los detalles del diseño, los grupos específicos incluidos en los programas y la escala eficiente de los mismos.

${ }^{20}$ Ver Tokman (2005). 
Según la teoría y evidencia internacional comentadas previamente, la principal limitación a la participación laboral de la mujer y los más jóvenes es la inexistencia de contratos de tiempo parcial. Si bien la última reforma incluye este tipo de contratos, queda pendiente la implementación de otras modalidades de contrato que se han aplicado con éxito en algunos países europeos. Éstas incluyen, primero, el desarrollo de empresas proveedoras de trabajo temporal, las que han tenido buenos resultados en otros países al reducir los costos de contratación por períodos breves - que muchas veces llevan a contratos informales en caso contrario-. Segundo, legislar sobre jornadas flexibles que permitan elegir la distribución de horarios dentro de un tiempo determinado, variando la intensidad de trabajo entre semanas o meses —-medida conocida como "banco de horas"-. Por supuesto, estas medidas tienen impactos positivos en todos los segmentos del mercado laboral, al incentivar la contratación ${ }^{21}$, aumentar la productividad del trabajo y reducir la variabilidad del empleo.

Una medida adicional para incentivar la participación de la mujer tiene relación con el financiamiento del cuidado infantil. La legislación actual exige a toda empresa con más de 20 mujeres la implementación de una sala cuna (o el financiamiento correspondiente), medida que podría desincentivar la contratación de mujeres, especialmente en empresas pequeñas en que se acercan a dicho número. En este contexto, financiar el costo con un impuesto compartido al trabajo de hombres y mujeres, de manera de reducir la discriminación femenina por este concepto, además de evaluar alternativas de pago compartido entre mujeres y empleadores, ayudarían a aumentar los bajos niveles de empleo femenino que presenta el país 22 .

Para el segmento más joven de la población, una medida adicional es reconsiderar la estructura del salario mínimo, incluyendo una diferenciación adicional por edades para el rango entre 18 y 24 años, además de ligar su reajuste a algún indicador macroeconómico objetivo y observable, como la tasa de desempleo de dicho segmento, las perspectivas económicas o alguna combinación de variables que tome en cuenta la coyuntura y permita

${ }^{21}$ El argumento teórico detrás del aumento en la contratación es relativamente simple: las empresas pueden sacar mayor provecho de su capacidad instalada si se les permite la alternación continua de "turnos" de trabajo, lo cual se ve limitado en ocasiones por la legislación actual. Adicionalmente, la medida permite a las empresas ajustarse a las necesidades estacionales de ciertas industrias, y condiciones cíclicas en general variando la cantidad de horas trabajadas en lugar de despedir trabajadores.

22 Otro aspecto que debiera ser explorado es el tratamiento tributario de los ingresos del segundo trabajador de la familia, de manera de incentivar la participación de la mujer en la generación de ingreso familiar. 
variar en forma procíclica. El propósito de esta medida sería permitir un mayor ajuste vía salarios, en lugar de desempleo, durante escenarios cíclicos recesivos. Finalmente, menores restricciones al uso de contratos a plazo fijo y/o contratos especiales “de prueba” permitirían a la empresa reducir el riesgo de contratación de dicho segmento.

\subsection{Capital humano: Fomentando la inversión en capacitación}

La literatura económica sugiere que existen fallas en el mercado del trabajo que llevan a una subinversión en capacitación respecto al nivel socialmente óptimo. En primer lugar, imperfecciones en el mercado de capitales impiden que muchos jóvenes financien programas de capacitación con cargo a ingresos futuros. Por otro lado, dado que los trabajadores pueden ser levantados por la competencia después de haber invertido en su formación general, las empresas no sólo asignan una menor cantidad de recursos en términos globales para capacitación, sino que sesgan su entrenamiento hacia aspectos específicos. Por último, el desconocimiento de los retornos potenciales de invertir en capacitación, así como la baja acreditación de las capacidades adquiridas, también explicarían un menor desarrollo del capital humano (Dolton, 1993).

Por estas razones, muchos países se han embarcado en programas de capacitación —-mediante subsidios estatales y/o beneficios tributarios a las empresas para capacitación en el trabajo—. No obstante, la literatura que evalúa dichos programas no es muy optimista respecto a sus beneficios, como muestran Friedlander et al. (1997) en su extensiva revisión. Sin embargo, la experiencia internacional permite sacar ciertas lecciones útiles. En particular, un programa adecuado debe incluir tanto subsidios estatales al desarrollo de habilidades generales —lectura, habilidades matemáticas básicas - como incentivos especiales a la capacitación realizada por las empresas.

Propuestas adicionales incluyen aumentar el monto de gastos de capacitación que se puede descontar del pago de indemnizaciones, así como la iniciativa de crear una certificación estatal de las habilidades adquiridas en los distintos programas de capacitación, ya que en la medida en que el capital humano se hace más tangible, aumentan los incentivos para invertir en él. En efecto, Lynch (1993) sugiere que una medida similar aplicada en Alemania explicaría una mejor disposición de los jóvenes a invertir en capacitación. 


\section{Conclusiones}

La teoría y la evidencia económica de numerosos estudios del mercado laboral sugieren que las regulaciones e instituciones juegan un rol importante en su funcionamiento. En efecto, a través del impacto en la rotación, la tasa natural de desempleo, la productividad del trabajo y la participación laboral de distintos grupos, las regulaciones e instituciones afectan en forma importante al crecimiento económico y al bienestar de los individuos. El desafío principal de los economistas y encargados de política económica radica en diseñar sistemas de protección valorados por los trabajadores, que afecten lo menos posible a la eficiencia de este mercado y corrijan las principales distorsiones existentes.

El mercado laboral en Chile se caracteriza por una tasa de desempleo relativamente alta y una baja participación del segmento de mujeres y jóvenes con relación al contexto internacional, tanto en economías desarrolladas como en países en transición. Entre las instituciones legales que podrían afectar a dichas variables se encuentran los altos costos de indemnizaciones y gastos judiciales que genera la terminación de contratos, lo que desincentiva tanto la contratación como la destrucción de puestos de baja productividad, reduciendo el potencial de crecimiento económico. Por otro lado, la rigidez de los contratos vigentes, así como el alto nivel del salario mínimo, serían candidatos importantes para explicar las bajas tasas de participación de los grupos mencionados.

La protección frente al desempleo ha avanzado con el seguro de cesantía implementado en la línea de las recomendaciones teóricas y los ejemplos de experiencias internacionales recientes. Su diseño en Chile se acerca bastante al sistema ideal dentro de una sociedad que valora el bienestar durante el desempleo, pero que tiene restricciones importantes en los montos que puede destinar el gobierno a administrar este tipo de seguridad social. En efecto, el perfil de pagos decreciente en el tiempo y el hecho de que los fondos provienen de una cuenta de capitalización individual corrigen la principal falla de incentivos de sistemas similares en Europa. La principal tarea pendiente es profundizar el seguro de desempleo en reemplazo del pago de indemnizaciones.

La flexibilidad en horarios, contratos y sistema de remuneraciones también apunta hacia un mercado laboral más acorde con las necesidades de empresas y trabajadores, con prometedores efectos en participación laboral, empleo y productividad. Adicionalmente, medidas que permitan diferenciar y moderar el ajuste de salarios mínimos de acuerdo con las condiciones macroeconómicas imperantes reducirían en escenarios recesivos las 
altas tasas de desempleo de la población más joven y de los menos capacitados. Por último, existen diversas medidas que pueden tomarse de manera de reducir las distorsiones que llevan a subinvertir en actividades de capacitación, lo cual mejoraría el nivel de capital humano, la productividad del trabajo y el crecimiento económico.

Uno de los principales desafíos adicionales se refiere a cómo mejorar la coordinación y comunicación entre empresas y sindicatos, factor determinante al explicar las diferencias internacionales en la tasa de desempleo y su persistencia ${ }^{23}$. Los países que han sido exitosos en lograr moderación en las negociaciones colectivas han logrado reducir las tasas de desempleo en el último tiempo. Las explicaciones que hay detrás de estas diferencias entre países, y la factibilidad de tomar medidas al respecto para superarlas es un tema con mucho potencial de desarrollo en la literatura económica, pero lamentablemente con pocas respuestas por el momento.

\section{REFERENCIAS}

Albagli, E., P. García y J. Restrepo: “Assessing the Flexibility of the Labor Market in Chile: An International Perspective”. En A. Tokman y J. Restrepo (eds.): Labor Markets and Institutions. Banco Central de Chile, 2005.

Bertola, G.: "Job Security, Employment and Wages". En European Economic Review, 34 (1990), pp. 851-886.

Bertola, G.: "Distribution, Efficiency and Labor Market Regulation; Theory, OECD, Latin America”. En A. Tokman y J. Restrepo (eds.): Labor Markets and Institutions. Banco Central de Chile, 2005.

Besley, T. y R. Burges: "Can Labor Regulation Hinder Economic Performance?: Evidence From India”. En The Quarterly Journal of Economics, Vol. 119 (1) (2004), pp. 91-134.

Blanchard, O. y L. Katz: "What we Know and do not Know about the Natural Rate of Unemployment”. En Journal of Economic Perspectives, 11 (1997), pp. 51-72.

Blanchard, O. y A. Landier: "The Perverse Effects of Partial Labor Market Reforms: Fixed-Term Contracts in France”. En Economic Journal, 112 (480) (2002), pp. 214-244.

Blanchard, O. y P. Portugal: "What Hides Behind an Unemployment Rate: Comparing Portuguese and U. S. Unemployment”. NBER Working Paper № 6636, 1998.

Blanchard, O. y T. Phillipon: "The Decline in Rents, and the Rise and Fall of European Unemployment”. MIT, mimeo, 2003.

Blanchard, O. y T. Phillipon: "The Quality of Labor Relations and Unemployment”. MIT Working Paper No 04-25, 2004.

Blanchard, O., y J. Tirole: "Designing Labor Market Institutions". En A. Tokman y J. Restrepo (eds.): Labor Markets and Institutions. Banco Central de Chile, 2005.

${ }^{23}$ Ver Blanchard y Phillipon (2004). 
Blundell, R. y M. Costas: “Active Labour Market Policy vs Employment Tax Credits: Lessons from Recent UK Reforms”. Institute for Labour Market Policy Evaluation, Working Paper 2002: 1, 2002.

Botero, J., S. Djankov, R. La Porta, F. Lopez de Silanes y A. Shleifer: "The Regulation of Labor”. NBER Working Paper 9756, 2003.

Bravo, D. y J. Vial: "La Fijación del Salario Mínimo en Chile: Elementos para una Discusión”. Colección Estudios CIEPLAN N 45, 1997, pp. 117-151.

Bravo, D. y D. Contreras: "Is There any Relationship Between Minimum Wage and Employment?”. Documento de Trabajo No 157 (2001), Departamento de Economía, Facultad de Ciencias Económicas, Universidad de Chile, Santiago.

Brown, J. y J. Earle: “Job Reallocation and Productivity Growth Under Alternative Economic Systems and Policies: Evidence from the Soviet Transition”. Upjohn Institute Staff Working Paper $\mathrm{N}^{\circ}$ 02-88, 2002.

Caballero, R., E. Engel y A. Micco: "Microeconomic Flexibility in Latin America”. En A. Tokman y J. Restrepo (eds.): Labor Markets and Institutions. Banco Central de Chile, 2005.

Caballero, R. y M. Hammour: "The Fundamental Transformation in Macroeconomics”. En American Economic Review, Vol. 86, N 2, 1996, pp. 181-186.

Caballero, R. y M. Hammour: "Jobless Growth: Appropriability, Factor Substitution, and Unemployment”. Carnegie-Rochester Conference Series on Public Policy 48, 1998, pp. 51-94.

Calderón, C. y A. Chong: "Are Labor Market Regulations an Obstacle for Long-Term Growth?”. En A. Tokman y J. Restrepo (eds.): Labor Markets and Institutions. Banco Central de Chile, 2005.

Calmfors, L. y J. Driffill: "Bargaining Structure, Corporativism and Macroeconomic Performance”. En Menil y Portes (eds.), Economic Policy: a European Forum, Vol. 3, $\mathrm{N}^{\circ} 1,1988$.

Calmfors, L., A. Forslund y M. Hemström: Seminar Paper No 700. Stockholm University, Institute for International Economic Studies, 2002.

Card, D. y A. Krueger: Myth and Measurement. The New Economics of the Minimum Wage on Employment and Unemployment. Princeton: N. J. Princeton University Press, 1995.

Cowan, K., A. Micco, A. Mizala, C. Pages y P. Romaguera: "Un Diagnóstico del Desempleo en Chile”. Mimeo, IADB, 2003.

Dolado, J., M. Jansen y J. Jimeno: “On the Effects of Targeted Employment Policies". En A. Tokman y J. Restrepo (eds.): Labor Markets and Institutions. Banco Central de Chile, 2005.

Dolton, P.: “The Economics of Youth Training in Britain”. En The Economic Journal, Vol. 103, No 420 (1993), pp. 1261-1278.

Forteza, A. y M. Rama: "Labor Market Rigidity and the Success of Economic Reforms across More than One Hundred Countries”. Banco Mundial, mimeo, 2002.

Friedlander, D., D. Greenberg y P. Robins: "Evaluating Government Training Programs for the Economically Disadvantaged”. En Journal of Economic Literature, Vol. 35, No 4 (1997), pp. 1809-1855.

Ham, J. y S. Rea: "Unemployment Insurance and Male Unemployment in Canada". En Journal of Labor Economics, 5 (1987), pp. 325-353. 
Heckmann, J. y C. Pagés: "The Cost of Job Security Regulations: Evidence from Latin American Labor Markets”. NBER. Working Paper N 7773, 2000.

Holmes, T.: "The Effects of State Policies on the Location of Manufacturing: Evidence From State Borders”. En Journal of Political Economy, 106 (1998), pp. 667705.

Hopenhayn, H.: "Labor Market Policies and Employment Duration: The Effects of Labor Market Reform in Argentina”. Research Network Working Paper R-407, Banco Inter-Americano de Desarrollo, 2001.

Jadresic, E.: "What Type of Contracts Underlie Aggregate Wage Dynamics?" IMF, Working Paper 97/67, 1997.

Jimeno, J. y D. Rodríguez-Palenzuela: "Youth Unemployment in the OECD: Demographic Shifts, Labor Market Institutions, and Macroeconomic Shocks”. Working Paper 2002-15. Madrid: Fundación de Estudios de Economía Aplicada, 2002.

Kim, J. y A. Campagna: "Effects of Flexitime on Employee Attendance and Performance: A Field Experiment”. En The Academy of Management Journal, Vol. 24, $\mathrm{N}^{\circ} 4$ (1981), pp. 729-741.

Kugler, A.: "The Impact of Firing Costs on Turnover and Unemployment: Evidence from the Colombian Labor Market Reform”. Mimeo, 2000.

Kugler, A., J. Jimeno y V. Hernanz: "Employment Consequences of Restrictive Employment Policies: Evidence from Spanish Labor Market Reforms". Working Paper 2003-14. Madrid: Fundación de Estudios de Economía Aplicada, 2003.

Lindbeck, A. y D. Snower: "Union Activity, Unemployment Persistence and WageEmployment Ratchets”. En European Economic Review, 31 (1987), pp. 157167.

Lynch, L.: "The Economics of Youth Training in the United States" En The Economic Journal, Vol. 103, № 420 (1993), pp. 1292-1302.

MacLeod, W. y J. Malcomson: "Investments, Holdup, and the Form of Market Contracts”. En The American Economic Review, Vol. 83, No 4 (1993), pp. 811-837.

Martínez, C., G. Morales y R. Valdés: “Cambios Estructurales en la Demanda por Trabajo en Chile”. En Economía Chilena, 4 (2) (2001): pp. 5-25.

Meyer, B.: "Unemployment Insurance and Unemployment Spells". En Econometrica, Vol. 58, N ${ }^{\circ} 4$ (1990), pp. 757-782.

Nunziata, L. y S. Staffolani: "The Employment Effects of Short-Term Contract Regulations in Europe”. Mimeo, 2001.

OECD: Employment Outlook. 1999.

OECD: "Labor Force Participation of Groups at the Margin of the Labor Market: Past and Future Trends and Policy Challenges”. Economics Department Working Paper, OECD, 2003a.

OECD: Economic Surveys. Chile 2003. 2003b.

Paredes, E. y A. Sanhueza: "Minimum Wages and School Drop-outs in Chile". Mimeo, 1996.

Rama, M. y R. Artecona: “A Database of Labor Market Indicators Across Countries”. Mimeo. Washington, DC: Banco Mundial, 2002.

Shapiro, C. y J. Stiglitz: "Equilibrium Unemployment as a Worker Discipline Device”. En The American Economic Review, Vol. 74, No 3 (1984), pp. 433-444. 
Siebert, H.: "Labor Market Rigidities: At the Root of Unemployment in Europe”. Journal Economic Perspectives, 11 (3) (1997), pp. 37-54.

Tokman, A.: "Participación Laboral en Chile”. Manuscrito, Banco Central de Chile, 2005. 